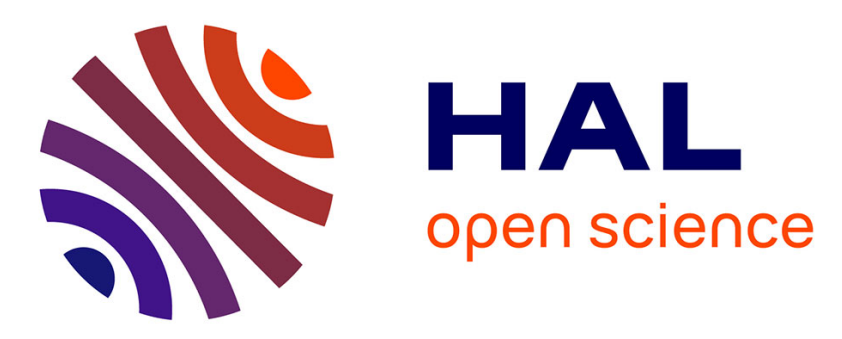

\title{
Impact of massive parallelization on two-photon absorption micro- and nanofabrication
}

\author{
Fabian Hilbert, Jonas Wiedenmann, Benedikt Stender, Willi Mantei, Ruth
}

Houbertz, Quentin Carlier, Luis Perez Covarrubias, Kevin Heggarty, Caroline Arnoux, Cyrille Monnereau, et al.

\section{To cite this version:}

Fabian Hilbert, Jonas Wiedenmann, Benedikt Stender, Willi Mantei, Ruth Houbertz, et al.. Impact of massive parallelization on two-photon absorption micro- and nanofabrication. SPIE LASE 2020, Feb 2020, San Francisco, CA, United States. pp.1127105, 10.1117/12.2545788 . hal-03104057

\section{HAL Id: hal-03104057 https://imt-atlantique.hal.science/hal-03104057}

Submitted on 26 May 2021

HAL is a multi-disciplinary open access archive for the deposit and dissemination of scientific research documents, whether they are published or not. The documents may come from teaching and research institutions in France or abroad, or from public or private research centers.
L'archive ouverte pluridisciplinaire HAL, est destinée au dépôt et à la diffusion de documents scientifiques de niveau recherche, publiés ou non, émanant des établissements d'enseignement et de recherche français ou étrangers, des laboratoires publics ou privés. 


\title{
Impact of massive parallelization on two-photon absorption micro- and nanofabrication
}

\author{
Fabian Hilbert*a, Jonas Wiedenmann ${ }^{\text {a }}$, Benedikt Stender ${ }^{\mathrm{a}}$, Willi Mantei ${ }^{\mathrm{a}}$, Ruth Houbertz ${ }^{\mathrm{a}}$, Quentin \\ Carlier $^{\mathrm{b}}$, Luis Perez Covarrubias ${ }^{\mathrm{b}}$, Kevin Heggarty ${ }^{\mathrm{b}}$, Caroline Arnoux ${ }^{\mathrm{c}}$, Cyrille Monnereau ${ }^{\mathrm{c}}$, Patrice \\ Baldeck $^{\mathrm{c}}$ \\ ${ }^{a}$ Multiphoton Optics GmbH, Friedrich-Bergius-Ring 15, 97076 Wuerzburg, Germany; ${ }^{\mathrm{b}} \mathrm{IMT}$ - \\ Atlantique, Optics Department, Technopole Brest-Iroise, 29285, Brest, France; 'Université de Lyon, \\ ENS de Lyon, CNRS UMR 5182, Université Claude Bernard Lyon 1, Laboratoire de Chimie, Lyon, \\ France
}

\begin{abstract}
The use of two-photon absorption (TPA) for polymerization, also known as 3D Lithography, Direct Laser Writing, or High-Precision 3D Printing is gaining increasing attraction in industrial fabrication of micro- and nanostructures. Mainly due to its vast freedom in design and high-resolution capabilities, TPA enables the fabrication of designs which are not feasible or far too complicated to be achieved with conventional fabrication methods. TPA is a scanning technology and fabrication in 3D requires axial overwritings. High industrial throughput fabrication can be achieved by intelligent fabrication strategies combined with an excellent material basis. Further boosting the throughput can be achieved by multispot exposure strategies. In this paper, massive parallelization is demonstrated which was realized by using a beam splitting diffractive optical element (DOE). Simultaneous fabrication using commercially available acrylate-based hybrid resin with 121 parallel focal spots arranged as $11 \times 11$ array is reported. Structures fabricated by a single laser beam and by 121 parallel beams are compared to each other with regard to shape and polymerization threshold. It was found that polymerization is strongly increased when parallel beams are used, especially for the central beams. As a result, polymerization threshold is lower in the center of the 11 x 11 array compared to the edges of the array. Furthermore, structures at the center of the 11 x 11 array are bigger compared to structures at the edges of the array when assigning equal intensity to all diffracted beams. These results are attributed to diffusion of photo initiators, quenchers, and radicals.
\end{abstract}

Keywords: two-photon absorption, two-photon polymerization, laser direct writing, diffractive optical element, parallelization

\section{INTRODUCTION}

The use of two-photon absorption (TPA) based polymerization, also known as 3D Lithography, Direct Laser Writing, or High-Precision 3D Printing is gaining increasing attraction in industrial fabrication of micro- and nanostructures. TPA enables the fabrication of complex 3D designs which are not feasible or far too complicated to be achieved with conventional fabrication methods ${ }^{1-6}$. The technique allows fabrication of real $3 \mathrm{D}$ structures with resolution typically down to $100 \mathrm{~nm}$ feature size with vast freedom for design. TPA is a scanning technology and fabrication in 3D requires axial overwritings. High industrial throughput fabrication can be achieved by intelligent fabrication strategies combined with an excellent material basis. Further boosting the throughput can be achieved by multi-spot exposure strategies. For the generation of multiple focal spots Spatial Light Modulators (SLM) and Diffractive Optical Elements (DOE) have been used in laser microfabrication ${ }^{7-12}$. Even microlens arrays were successfully applied for parallel TPA fabrication, but this requires an accurate flat top beam across the area of the microlens array ${ }^{13}$. All these parallelization techniques have in common, that they scale the fabrication throughput by the number of focal spots. In the same time a single structure is produced with a single beam, multiple structures of the same type can be fabricated when multiple focal spots are used. An SLM is an active device and allows on-the-fly switching of the diffraction pattern. This enables the use of the same SLM in different applications with individual spot patterns. Since a DOE is a passive optical element, it generates a fixed pattern and cannot be simply adapted on-the-fly. However, an SLM usually shows low diffraction efficiency and generates a strong zeroth order. Oftentimes a big effort is necessary to block or to remove this zeroth order from the optical 
path $^{8,11,14,15}$. With a high quality DOE, the zeroth order can be avoided much better than with an SLM. Efficiency is therefore higher when high quality DOEs are used compared to SLMs.

In this work, we demonstrate massively parallelized TPA-based polymerization with 121 parallel beams, generated by a DOE. We compare structures that were fabricated with a single beam to structures fabricated with parallel beams. It is shown that fabrication time can be reduced by a factor of 121, equal to the number of parallel beams. At the same time, the applied laser power has to be increased only by a factor of 56 to 80 , due to a proximity effect which lowers the polymerization threshold.

\section{MATERIALS AND METHODS}

All structures fabricated presented in this work were made from the commercially available inorganic-organic resin OrmoComp $^{\circledR}$ (micro resist technology GmbH, Berlin, Germany). This material is well known and valued in microfabrication for its excellent optical properties ${ }^{16,17}$. The resin was formulated with 2 wt.- $\%$ of a photoinitiator of an Irgacure series ([1-[9-ethyl-6-(2-methylbenzoyl)carbazol-3-yl]ethylideneamino] acetate) to generate a low polymerization threshold. Solid structures were fabricated via two-photon absorption (TPA) induced polymerization using femtosecondlaser pulses. For this works, the liquid resin is enclosed tightly between two glass slides and a spacer, as shown in Figure 1(b)). As a result, no interaction is possible between the resin and air during the fabrication, preventing radical-scavenging.

TPA fabrication was performed using a standard LithoProf3D ${ }^{\circledR}$ equipment (Multiphoton Optics GmbH, Würzburg, Germany). For parallel fabrication, an additional diffractive optical element (DOE) was introduced into the optical path in front of the focusing objective. A schematic sketch of the optical path is shown in Figure 1(a). As laser source, a pulsed fs laser with a central wavelength of $522 \mathrm{~nm}$ (FWHM $2 \mathrm{~nm}$ ), a repetition rate of $63 \mathrm{MHz}$, and a pulse duration (FWHM) of $250 \mathrm{fs}$ is used. Focusing of the laser was done with an oil immersion microscope objective Plan-Apochromat 100x/ NA 1.4 (Carl Zeiss Microscopy GmbH, Jena, Germany). The field of view (FoV) of the applied objective is $250 \mu \mathrm{m}$ according to the manufacturer. All average laser powers used for fabrication (see sections 2.2 and 2.1) were measured before the laser beam enters the DOE (or the objective in case of single beam fabrication). The average power used for fabrication was adjusted on purpose as described below. Transmission of the objective for the applied wavelength $522 \mathrm{~nm}$ is about 92 $\%$. The structures were fabricated using galvo-scanners for $\mathrm{X}-\mathrm{Y}$ motion and an air bearing stage for $\mathrm{Z}$ motion.

(a)

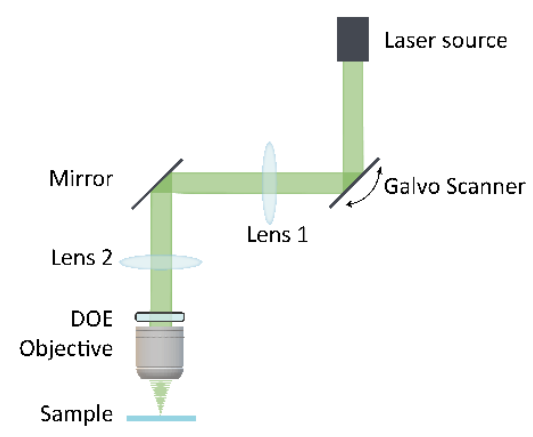

(b)

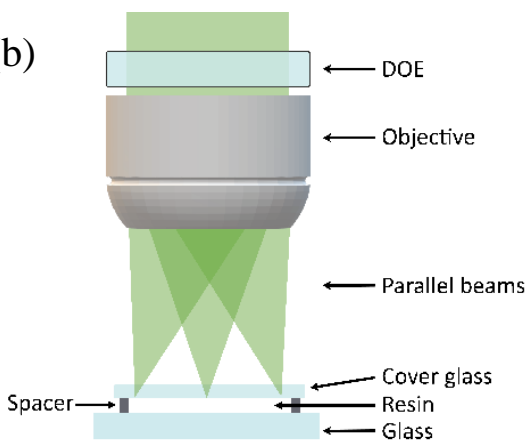

Figure 1. (a) Laser setup used for TPA single-beam and multi-beam fabrication. A 4 f system (Lens 1, Lens 2 ) is used to project the motion of the galvo-scanner mirrors into the rear aperture of the objective. The beam splitting DOE is inserted directly before the objective as depicted for parallel fabrication with 121 beams. The DOE is removed for single beam fabrication. (b) Schematic close-up view of DOE, objective, and sample. The DOE splits the incoming beam into multiple beams, which are focused at separate positions in the focal plane.

After TPA fabrication, all structures were immersed in a mixture of methyl isobutyl ketone and isopropanol 1:3 (weight ratio) for development. For characterization of the fabricated structures a scanning electron microscope (SEM) (Hitachi) and a laser scanning microscope (LSM) (Olympus) were used. 


\subsection{Single beam fabrication}

Cuboid structures with edge lengths $d=2.88 \mu \mathrm{m}, 5.76 \mu \mathrm{m}$, and $8.64 \mu \mathrm{m}$ were fabricated with a single focused laser beam as a reference for comparison with parallel fabrication (cf. section 2.2). Hatch and slice distances of $0.1 \mu \mathrm{m}$ were chosen to create the volume of each structure. Average laser powers ranging from 100 to $850 \mu \mathrm{W}$ (increment $150 \mu \mathrm{W}$ ) were applied for fabrication of the single structures.

\subsection{Parallel fabrication}

For fabrication with 121 parallel laser beams, a custom designed binary DOE was used. The DOE itself was fabricated by maskless microlithography using Microposit ${ }^{\mathrm{TM}} \mathrm{S} 1800$ resist on a glass substrate as described in prior work ${ }^{18}$. The DOE generates an array of $11 \times 11$ equally spaced spots with a pitch p of $9.3 \mu \mathrm{m}$ in the focal plane of the objective. The central spot of the array coincides with the direction of the non-diffracted light, i.e. the direction of the zeroth order.

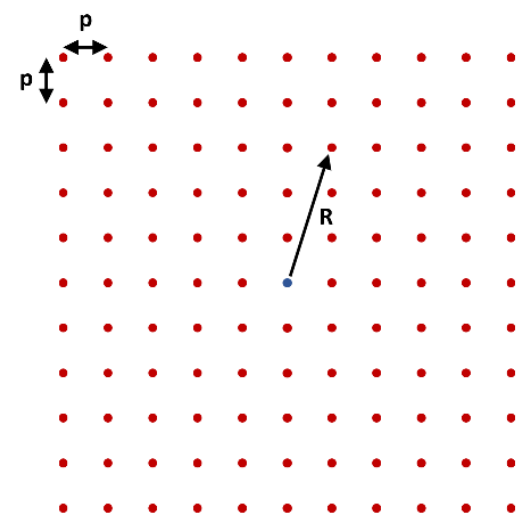

Figure 2. Pattern design for the beam splitting DOE. An array of $11 \mathrm{x} 11$ focal spots with homogeneous intensity distribution is generated in the focal plane of the objective, and 121 parallel beams are generated. The pitch $\mathrm{p}$ between the focal spots is $9.3 \mu \mathrm{m}$, resulting in a total expansion of the focal spots over an area of $93 \mu \mathrm{m}$ x $93 \mu \mathrm{m}$. The central spot (blue) coincides with the direction of the zeroth order diffracted light. The distance of each spot from the central spot is denoted as R.

Introducing the DOE directly before the microscope objective is done to make sure that all diffracted beams fully enter the objective rear aperture. If the DOE was placed further away from the rear aperture, the beams would have diverged and might have not fully entered the objective rear aperture. This could have resulted in inhomogeneous intensity across the beams. The focal spots which are farthest away from the central spot span a total distance of $131.5 \mu \mathrm{m}$ (corresponding to the diagonal of the spot array in Figure 2), which is well below the size of the FoV $(250 \mu \mathrm{m})$. The applied binary DOE was optimized for uniformity, resulting in a simulated efficiency of $75 \%$ for the first order diffraction spots. The actual overall efficiency, i.e. the power of first order diffraction spots/total incident power of the DOE was characterized to be $66 \%$.

Cuboids with square bases of three different sizes (edge lengths d) were fabricated with the beam splitting DOE. All cuboids were designed to constant height $\mathrm{h}=5 \mu \mathrm{m}$. The edge length $\mathrm{d}$ was chosen in order to generate 1D fill factors of the multispot array $\mathrm{f}=\mathrm{d} / \mathrm{p}$ of 31,62 , and $93 \%$, resulting in $\mathrm{d}_{31 \%}=2.88 \mu \mathrm{m}, \mathrm{d}_{62 \%}=5.76 \mu \mathrm{m}$ and $\mathrm{d}_{93 \%}=8.64 \mu \mathrm{m}$, respectively. Pyramids with a base edge length of $4 \mu \mathrm{m}$ were also fabricated.

Structures were fabricated in parallel with total average laser powers of $20,17,14,11$, and $8 \mathrm{~mW}$. Since the total diffraction efficiency of the DOE was about $66 \%$, the effective power for each of the 121 beams was $109,93,76,60$, and $44 \mu \mathrm{W}$, respectively. The structures were fabricated by filling the entire volume of the structures with hatches of $0.1 \mu \mathrm{m}$ distance and slices of $0.1 \mu \mathrm{m}$ distance. 


\section{RESULTS}

\subsection{Single beam fabrication}

Cuboids with a square base of edge length of $2.88,5.76$, and $8.64 \mu \mathrm{m}$ fabricated with average laser power from 250 to 800 $\mu \mathrm{W}$ are shown in Figure 3. No structures were obtained from $100 \mu \mathrm{W}$ mean laser power.

(a)

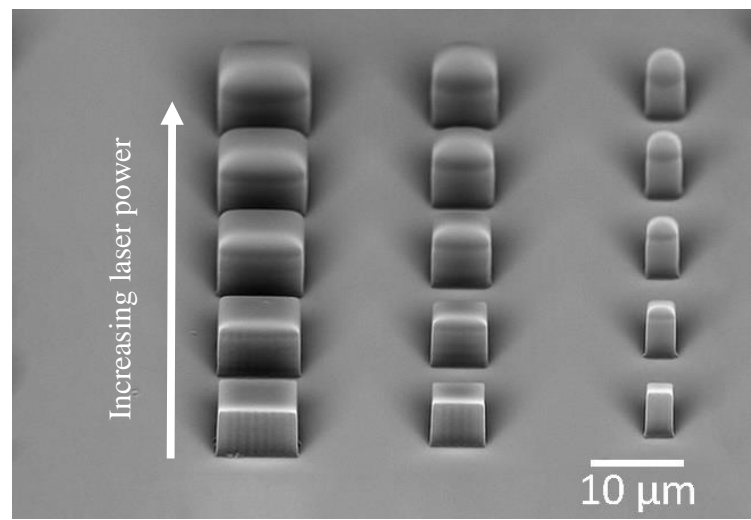

(b)

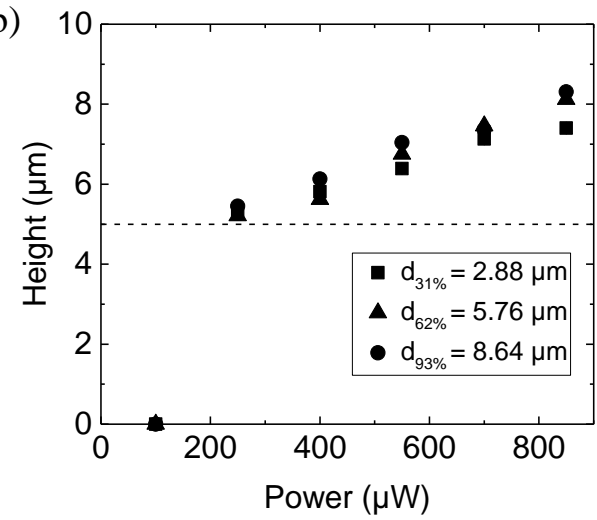

Figure 3. (a) Cuboids with a square base of edge length $\mathrm{d}_{93} \%=8.64 \mu \mathrm{m}$ (left), $\mathrm{d}_{62} \%=5.76 \mu \mathrm{m}$ (center), and $\mathrm{d}_{31} \%=2.88 \mu \mathrm{m}$ (right), fabricated with a single laser beam. The power was increased from $250 \mu \mathrm{W}$ (bottom row) to $850 \mu \mathrm{W}$ (upper row) with an increment of $150 \mu \mathrm{W}$. No structures were obtained for an average laser power of $100 \mu \mathrm{W}$. The cuboid shape is maintained very well for the structure fabricated with $250 \mu \mathrm{W}$ (bottom row) with continuously increasing round shape for increasing power. (b) Height of the cuboids (determined in the center of each structure) as a function of the average laser power. The design height of $5 \mu \mathrm{m}$ is indicated by the dashed line. The height correlates with the applied power.

The height of structures fabricated with a single beam increases with increasing power due to an elongation of the focal volume along the optical axis (z direction) (see Figure 3). In contrast, there is no significant difference in height among cuboids of different edge length but identical average power.

\subsection{Parallel fabrication}

Structures were fabricated with 11 x 11 parallel beams, generated by the DOE. Figure 4 shows pyramids fabricated in parallel with an edge length of $4 \mu \mathrm{m}$ and a height of $12 \mu \mathrm{m}$.

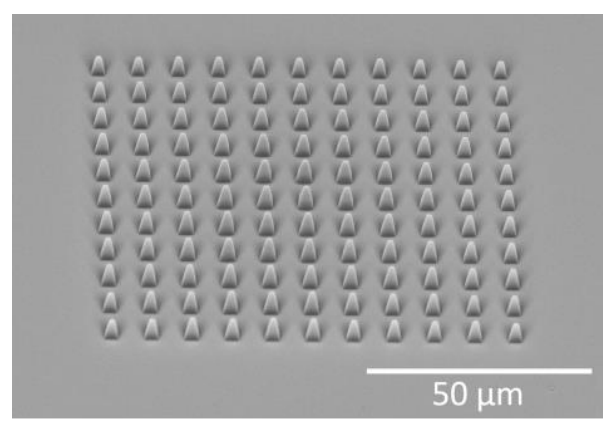

Figure 4. Pyramids, parallelly fabricated with an $11 \times 11$ beam splitting DOE using $44 \mu \mathrm{W}$ average laser power per beam. The base edge length is $4 \mu \mathrm{m}$, the design height is $12 \mu \mathrm{m}$. The total fabrication time was $70 \mathrm{~s}$.

Furthermore, cuboid structures were fabricated with 11 x 11 parallel beams, as shown in Figure 5. It appeared that with decreasing power, an increasing number of structures is missing at the edges of the array. The number of stable structures 
fabricated with a given average laser power is increased for larger structures (i.e. higher fill factor), as shown in Figure 5. For a given structure size, an increase in laser power leads to over-exposure mainly in the center of the array, while structures at the edges of the array are still well separated. When the average laser power is reduced, structures at the edges of the array disappear, while structures in the center of the array are still well maintained (see Figure 6).
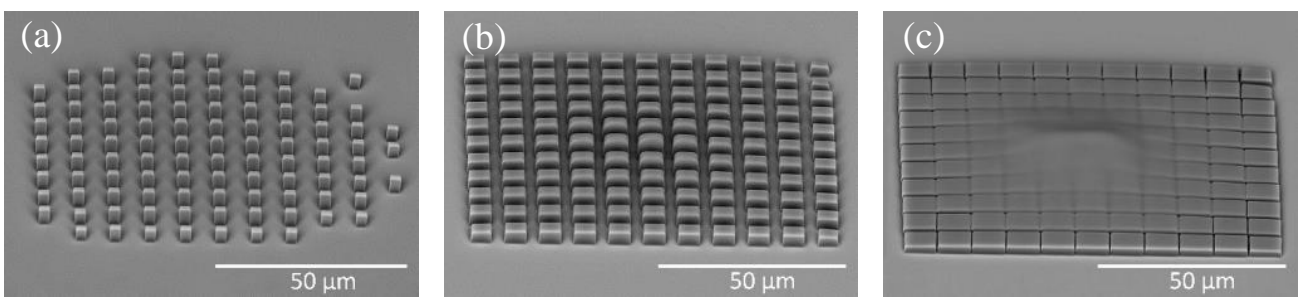

Figure 5. Cuboids fabricated in parallel with an $11 \times 11$ beam array with an average power of $76 \mu \mathrm{W}$ per beam. Cuboids with a design edge length of (a) $2.88 \mu \mathrm{m}$ (fill factor: $31 \%$ ), (b) $5.76 \mu \mathrm{m}$ (fill factor: $62 \%$ ), and (c) $8.64 \mu \mathrm{m}$ (fill factor: $93 \%$ ).

Although all structures were fabricated with equal power, some of the outer structures are missing for the smallest edge length (Figure 5 (a)), while all structures are present for larger edge lengths (Figure 5 (b) and (c)). With the largest edge length, structures in the center of the array merge to a single, large polymerized block. Cuboids with an edge length of 5.76 $\mu \mathrm{m}$ using an average laser power per beam of 76 and $60 \mu \mathrm{m}$ are shown in Figure 6 . All structures are present and well separated when using $76 \mu \mathrm{W}$ for fabrication. The size of the structures is varying, with the central structures slightly larger and decreasing sizes towards the outer areas. If a smaller average laser power $(60 \mu \mathrm{W})$ is used, some of the outer structures are missing (see also Table 1).
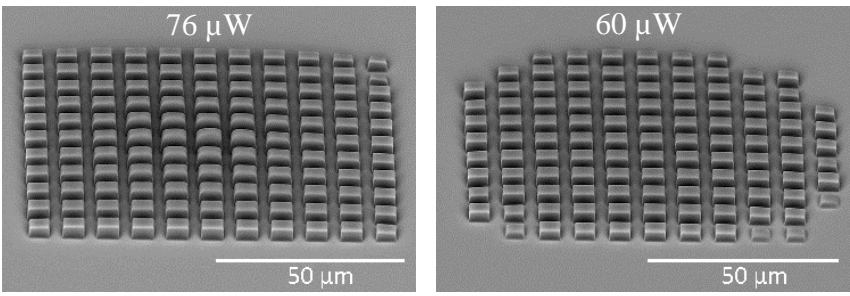

Figure 6. Cuboids with an edge length of $5.76 \mu \mathrm{m}$, fabricated with an average laser power per beam of (a) $76 \mu \mathrm{W}$ and (b) 60 $\mu \mathrm{W}$. All structures are present and well separated when using $76 \mu \mathrm{W}$. Some of the outer structures are lacking when using 60 $\mu \mathrm{W}$ (cf. Table 1).

Table 1 summarizes the results on the number of structures in dependence of the average laser power and the edge length, when using the $11 \times 11$ parallel beam array.

Table 1. Number of structures present after fabrication with 121 parallel beams (cf. Figure 5 and Figure 6) for average laser power per beam ranging from 44 to $109 \mu \mathrm{W}$ and cuboid edge lengths of 2.88, 5.76, and 8.64 $\mu \mathrm{m}$. Color code: Green: Structures from all 121 beams are existing. Yellow: Not all structures from 121 beams are existing. Orange: No structure existing.

\begin{tabular}{|c|c|c|c|}
\hline Power per beam $(\mu \mathrm{W})$ & Edge length $2.88 \mu \mathrm{m}$ & Edge length $5.76 \mu \mathrm{m}$ & Edge length $8.64 \mu \mathrm{m}$ \\
\hline 109 & 121 & 121 & 121 \\
\hline 93 & 118 & 121 & 121 \\
\hline 76 & 100 & 121 & 121 \\
\hline 60 & 22 & 110 & 113 \\
\hline 44 & 0 & 10 & 25 \\
\hline
\end{tabular}


The height of structures fabricated in parallel varies across the array. Figure 7 plots the structure heights as a function of $\mathrm{R}$ (defined in Figure 2) for the different edge lengths. Structures show a lower height with increasing R, i.e. they are lower the further away they are from the center of the array. For constant average laser power (i.e. same marker color in all subfigures of Figure 7), the structure height increases significantly with edge length of the cuboids, i.e. fill factor.

(a)

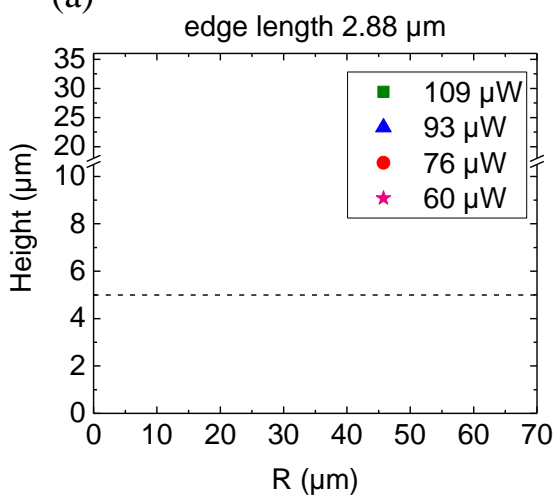

(b)

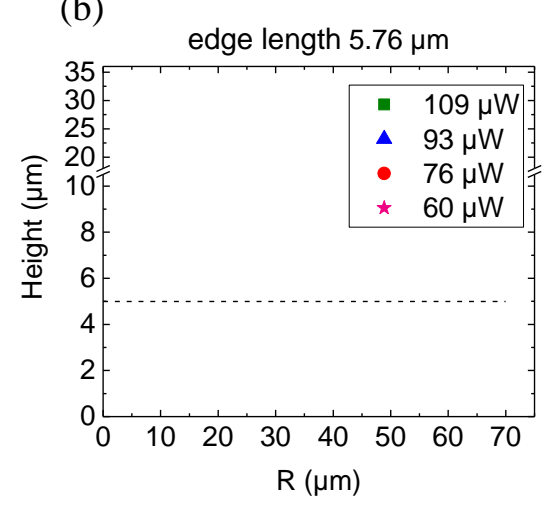

(c)

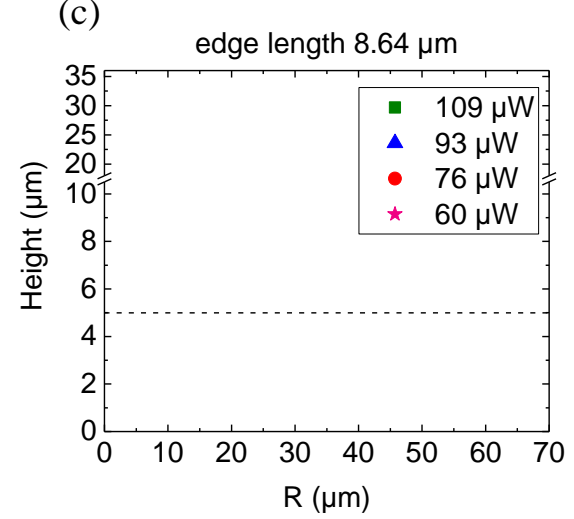

Figure 7. Structure heights of structures fabricated with $11 \mathrm{x} 11$ parallel beams as function of R (see Figure 2). The height is given for an effective power per beam from 60 to $109 \mu \mathrm{W}$ for cuboids with an edge length of (a) $2.88 \mu \mathrm{m}$, (b) $5.76 \mu \mathrm{m}$, and (c) $8.64 \mu \mathrm{m}$. The design height of $5 \mu \mathrm{m}$ is indicated by the dashed line. Each point indicates the mean over all $\mathrm{n}$ structures with identical $R$, where $n=4$ or $n=8$ for all $R>0$ and $n=1$ for $R=0$. Error bars are plotted, but smaller than the marker size for most points.

For each edge length, heights are given as a function of distance $\mathrm{R}$ from the central spot (see Figure 2) for a single power $(2.88 \mu \mathrm{m} \rightarrow 109 \mu \mathrm{W}, 5.76 \mu \mathrm{m} \rightarrow 76 \mu \mathrm{W}, 8.64 \mu \mathrm{m} \rightarrow 60 \mu \mathrm{W})$. For other powers, only the height in the center of the array $(\mathrm{R}=0)$ is given. No structures were obtained when using $60 \mu \mathrm{W}$ average power per beam in combination with edge length $2.88 \mu \mathrm{m}$. The time for parallel fabrication of an $11 \times 11$ array is equal to the time required for fabrication of a single structure with a single beam (see Table 2).

Table 2. Time for the parallel fabrication of 121 (11 x 11) cuboids with a given edge length. The same time is required for the fabrication of a single structure of equal size with a single beam.

\begin{tabular}{|l|l|}
\hline Edge length $(\boldsymbol{\mu m})$ & Fabrication time (s) \\
\hline 2.88 & 44 \\
\hline 5.76 & 171 \\
\hline 8.64 & 327 \\
\hline
\end{tabular}

\section{DISCUSSION}

Massively parallelized TPA fabrication is demonstrated exemplary for a factor of 121 by implementing an $11 \mathrm{x} 11$ beam splitting DOE into the fabrication system. The time for parallel fabrication of an entire $11 \mathrm{x} 11$ structure array was identical to the fabrication time of a single structure with a single beam. This means an increase of throughput by a factor of 121 . Prior works have used DMDs or SLMs for parallel TPA fabrication with usually only a few beams up to no more than 30 beams ${ }^{8,12,19,20}$. Compared to these prior works, no effort was necessary in this work to block the zeroth order beam due to the excellent design and fabrication of the DOE. Instead of blocking the zeroth order, we used a designed first order diffraction spot in the direction of the zeroth order beam for fabrication. Formanek et al. fabricated over 700 structures in parallel with TPA using a microlens array ${ }^{13,21}$. However, they had to shape their beam from Gaussian to flat top profile to illuminate the microlens array homogeneously. Since we use a DOE for beam splitting, there was no need for additional beam shaping. This allows easy switching between single beam and multi-beam fabrication by simply moving the DOE in and out the optical path. No further changes to the optical path were necessary to enhance the standard TPA LithoProf3D ${ }^{\circledR}$ equipment from single beam to multi-beam fabrication. 
The fabricated structures show some major differences when they were fabricated with a single beam compared to 121 parallel beams. In single beam serial fabrication, the structure height scales with the applied average laser power, while there is no major difference in height between structures of different edge length and equal power. In parallel fabrication, structures at the center of the array tend to become bigger than structures at the edges of the array, although they are exposed to the same power, thus seeing the same exposure dose. Furthermore, far less power is necessary per beam when fabricating in parallel compared to single beam exposure. With a single beam, an average power of $100 \mu \mathrm{W}$ was not enough to exceed the threshold to initiate organic cross-linking, resulting in stable polymerized structures, while for parallel fabrication solid structures were obtained with powers down to $44 \mu \mathrm{W}$ per beam. This shows that TPA-induced polymerization at one position in the resin is influenced by TPA-based polymerizations in its temporal and/or spatial proximity. This proximity effect can be explained by diffusion of photoinitiators, quenchers (such as oxygen) and radicals. Radicals may be generated in the focal volume of one beam and then diffuse towards other nearby focal volumes. The polymerization threshold is thus reduced the more beams are used in parallel and the smaller the gap is between neighboring structures. This hypothesis is supported by the fact that less power per beam is necessary for polymerization of the larger structures in the work, i.e. for larger fill factors. Furthermore, the polymerization threshold decreases most strongly in the center of the beam array, where more surrounding beams are present compared to the edges of the array. Hence, when using low laser power, structures are polymerized at the center of the array, but not at the edges of the array.

A spatial proximity effect for TPA-based polymerization was reported already for single beam fabrication ${ }^{22}$. The broadening of a TPA-fabricated single lines has been demonstrated in the presence of a second polymerizing line in close spatial and temporal proximity ${ }^{23}$. However, if the reason for proximity effect is diffusion, the strength of this effect may vary from material to material. Furthermore, it should vary with the distance of the parallel beams and number of total parallel beams. In our setup, no interaction between air and resin was possible during fabrication (see Figure 1 (b)). This prevents oxygen, which acts as a quencher, from diffusing into the material during fabrication. The amount of oxygen inside the material system is limited in our setup. In a different experimental setup, which allows interaction between the resin and air during the fabrication, there might be an increased amount of oxygen in the resin. In such setup there could be more radical quenching, which reduces the proximity effect.

Adjusting the intensity of the generated multiple focal spots by enhancement of the DOE design will be subject to future work. A reduction of intensity in the center of the beam array shows great promise for parallel TPA fabrication of identical structures with even more parallel beams which will be reported elsewhere.

\section{ACKNOWLEDGMENTS}

This work has received funding from the European Union's Horizon 2020 research and innovation program under grant agreement $\mathrm{N}^{\mathrm{o}} 780278$. The dissemination of results herein reflects only the author's view and the European Commission is not responsible for any use that may be made of the information it contains.

\section{REFERENCES}

[1] Kurselis, K., Kiyan, R., Bagratashvili, V. N., Popov, V. K. and Chichkov, B. N., “3D fabrication of all-polymer conductive microstructures by two photon polymerization," Opt. Express 21(25), 31029 (2013).

[2] Yu, H., Zhang, Q. and Gu, M., "Three-dimensional direct laser writing of biomimetic neuron structures," Opt. Express 26(24), 32111 (2018).

[3] Vangelatos, Z., Melissinaki, V., Farsari, M., Komvopoulos, K. and Grigoropoulos, C., "Intertwined microlattices greatly enhance the performance of mechanical metamaterials," Math. Mech. Solids 24(8), 2636-2648 (2019).

[4] Houbertz, R. and Steenhusen, S., "Two-photon polymerization of inorganic-organic hybrid polymers as scalable technology using ultra-short laser pulses," [Coherence and Ultrashort Pulse Laser Emission: InTech], 583-608 (2010).

[5] Steenhusen, S., Stichel, T., Houbertz, R. and Sextl, G., "Multi-photon polymerization of inorganic-organic hybrid polymers using visible or IR ultrafast laser pulses for optical or optoelectronic devices," Proc. SPIE 7591(Advanced Fabrication Technologies for Micro/Nano Optics and Photonics III), W. V. Schoenfeld, J. J. Wang, M. Loncar, and T. J. Suleski, Eds., 759114 (2010).

[6] Stichel, T., Hecht, B., Houbertz, R. and Sextl, G., "Two-photon polymerization as method for the fabrication of 
large scale biomedical scaffold applications," J. Laser Micro Nanoeng. 5(3), 209-212 (2010).

[7] Kelemen, L., Valkai, S. and Ormos, P., "Parallel photopolymerisation with complex light patterns generated by diffractive optical elements," Opt. Express 15(22), 14488 (2007).

[8] Zandrini, T., Shan, O., Parodi, V., Cerullo, G., Raimondi, M. T. and Osellame, R., "Multi-foci laser microfabrication of 3D polymeric scaffolds for stem cell expansion in regenerative medicine," Sci. Rep. 9(1), 19 (2019).

[9] Johnson, E. G., Poutous, M. K., Roth, Z. A., Srinivasan, P., Pung, A. J. and Yilmaz, Y. O., "Advanced fabrication methods for 3d meta-optics," Adv. Fabr. Technol. Micro/Nano Opt. Photonics IV 7927, 792706792706-792707 (2011).

[10] Winfield, R. J., Bhuian, B., O’Brien, S. and Crean, G. M., "Fabrication of grating structures by simultaneous multi-spot fs laser writing,” Appl. Surf. Sci. 253(19), 8086-8090 (2007).

[11] Obata, K., Koch, J., Hinze, U. and Chichkov, B. N., "Multi-focus two-photon polymerization technique based on individually controlled phase modulation," Opt. Express 18(16), 17193 (2010).

[12] Gittard, S. D., Nguyen, A., Obata, K., Koroleva, A., Narayan, R. J. and Chichkov, B. N., "Fabrication of microscale medical devices by two-photon polymerization with multiple foci via a spatial light modulator," Biomed. Opt. Express 2(11), 3167 (2011).

[13] Formanek, F., Takeyasu, N., Tanaka, T., Chiyoda, K., Ishikawa, A. and Kawata, S., "Three-dimensional fabrication of metallic nanostructures over large areas by two-photon polymerization," Opt. Express 14(2), 800 (2006).

[14] Yang, L., Li, J., Hu, Y., Zhang, C., Lao, Z., Huang, W. and Chu, J., "Projection two-photon polymerization using a spatial light modulator," Opt. Commun. 331, 82-86 (2014).

[15] Kuang, Z., Liu, D., Perrie, W., Edwardson, S., Sharp, M., Fearon, E., Dearden, G. and Watkins, K., "Fast parallel diffractive multi-beam femtosecond laser surface micro-structuring,” Appl. Surf. Sci. 255(13-14), 6582-6588 (2009).

[16] Houbertz, R., Wolter, H., Dannberg, P., Serbin, J. and Uhlig, S., "Advanced packaging materials for optical applications: bridging the gap between $\mathrm{nm}$-size structures and large-area panel processing," Proc. SPIE 6126, A. M. Earman and R. T. Chen, Eds., 612605 (2006).

[17] Stender, B., Hilbert, F., Dupuis, Y., Krupp, A., Mantei, W. and Houbertz, R., "Manufacturing strategies for scalable high-precision 3D printing of structures from the micro to the macro range," Adv. Opt. Technol. 8(3-4), 225-231 (2019).

[18] Kessels, M. V., El Bouz, M., Pagan, R. and Heggarty, K., "Versatile stepper based maskless microlithography using a liquid crystal display for direct write of binary and multilevel microstructures," J. Micro/Nanolithography, MEMS, MOEMS 6(3), 033002 (2007).

[19] Hu, Y., Chen, Y., Ma, J., Li, J., Huang, W. and Chu, J., "High-efficiency fabrication of aspheric microlens arrays by holographic femtosecond laser-induced photopolymerization," Appl. Phys. Lett. 103(14), 1-5 (2013).

[20] Geng, Q., Wang, D., Chen, P. and Chen, S. C., "Ultrafast multi-focus 3-D nano-fabrication based on two-photon polymerization," Nat. Commun. 10(1), 1-7 (2019).

[21] Sun, H. B. and Kawata, S., "Two-photon photopolymerization and 3D lithographic microfabrication," Adv. Polym. Sci. 170, 169-273 (2004).

[22] Saha, S. K., Divin, C., Cuadra, J. A. and Panas, R. M., "Effect of proximity of features on the damage threshold during submicron additive manufacturing via two-photon polymerization," J. Micro Nano-Manufacturing 5(3) (2017).

[23] Waller, E. H. and von Freymann, G., "Spatio-temporal proximity characteristics in 3D $\mu$-printing via multiphoton absorption," Polymers (Basel). 8(8), 1-13 (2016). 